Article

\title{
On the Generalization of a Class of Harmonic Univalent Functions Defined by Differential Operator
}

\author{
Aqeel Ketab AL-khafaji ${ }^{1,2, *} \mathbb{C}$, Waggas Galib Atshan ${ }^{3}$ and Salwa Salman Abed ${ }^{2}$ \\ 1 Department of Mathematics, College of Education for Pure Sciences, University of Babylon, \\ Babylon 51002, Iraq \\ 2 Department of Mathematics, College of Education for Pure Sciences-Ibn Al-Haytham, \\ The University of Baghdad, Baghdad 10071, Iraq; salwaalbundi@yahoo.com \\ 3 Department of Mathematics, College of Computer Science \& Information Technology, \\ The University of Al-Qadisiyah, Al Diwaniyah 58002, Iraq; waggashnd@gmail.com \\ * Correspondence: aqeelketab@gmail.com
}

Received: 30 October 2018; Accepted: 27 November 2018; Published: 7 December 2018

check for updates

\begin{abstract}
In this article, a new class of harmonic univalent functions, defined by the differential operator, is introduced. Some geometric properties, like, coefficient estimates, extreme points, convex combination and convolution (Hadamard product) are obtained.
\end{abstract}

Keywords: harmonic univalent function; coefficient inequality; extreme points; convex combination; Hadamard product

\section{Introduction}

A continuous function $f=u+i v$ is a complex-valued harmonic function in a complex domain $\mathbb{C}$ if both $u$ and $v$ are real harmonic. In any simply connected domain $\mathcal{B} \subset \mathbb{C}$, we can write $f=h+\bar{g}$, where $h$ and $g$ are analytic in $\mathcal{B}$. We call $h$ and $g$ are analytic part and co-analytic part of $f$ respectively. Clunie and Sheil-Small [1] observed that a necessary and sufficient condition for the harmonic functions $f=h+\bar{g}$ to be locally univalent and sense-preserving in $\mathcal{B}$ is that $\left|h^{\prime}(z)\right|>\left|g^{\prime}(z)\right|,(z \in \mathcal{B})$.

Denote by $S_{H}$ the family of harmonic functions $f=h+\bar{g}$, which are univalent and sense-preserving in the open unit disc $U=\{z \in \mathbb{C}:|z|<1\}$ where $h$ and $g$ are analytic in $\mathcal{B}$ and $f$ is normalized by $f(0)=h(0)=f_{z}(0)-1=0$. Then for $f=h+\bar{g} \in S_{H}$, we may express the analytic functions $h$ and $g$ as

$$
h(z)=z+\sum_{n=2}^{\infty} a_{n} z^{n}, g(z)=\sum_{n=1}^{\infty} b_{n} z^{n},\left|b_{1}\right|<1 .
$$

Note that $S_{H}$ reduces to the class of normalized analytic univalent functions if the co-analytic part of its members equals to zero.

Also, denote by $S_{\bar{H}}$ the subclass of $S_{H}$ consisting of all functions $f_{k}(z)=h(z)+\overline{g_{k}(z)}$, where $h$ and $g$ are given by

$$
h(z)=z-\sum_{n=2}^{\infty}\left|a_{n}\right| z^{n} \text { and } g_{k}(z)=(-1)^{k} \sum_{n=1}^{\infty}\left|b_{n}\right| z^{n},\left|b_{1}\right|<1 .
$$

In 1984 Clunie and Sheil-Small [1] investigated the class $S_{H}$, as well as its geometric subclass and obtained some coefficient bounds. Many authors have studied the family of harmonic univalent function (see References [2-7]). 
In 2016 Makinde [8] introduced the differential operator $F^{k}$ such that

$$
F^{k} f(z)=z+\sum_{n=2}^{\infty} C_{n k} z^{n}
$$

where

$$
C_{n k}=\frac{n !}{|n-k| !}, F^{k} f(z)=z^{k}\left[z^{-(k-1)}+\sum_{n=2}^{\infty} C_{n k} z^{n}\right], k \in \mathbb{N}_{0}=\mathbb{N} \cup\{0\}
$$

and

$$
F^{0} f(z)=f(z), F^{1} f(z)=z+\sum_{n=2}^{\infty} C_{n 1} z^{n}
$$

Thus, it implies that $F^{k} f(z)$ is identically the same as $f(z)$ when $k=0$. Also, it reduced the first differential coefficient of the Salagean differential operator when $k=1$.

For $f=h+\bar{g}$ given by Equation (1), Sharma and Ravindar [9] considered the differential operator which defined by Equation (3) of $f$ as

$$
F^{k} f(z)=F^{k} h(z)+(-1)^{k} \overline{F^{k} g(z)}, k \in \mathbb{N}_{0}=\mathbb{N} \cup\{0\}, z \in \mathbb{C},
$$

where

$$
F^{k} h(z)=z+\sum_{n=2}^{\infty} C_{n k} a_{n} z^{n}, F^{k} g(z)=\sum_{n=1}^{\infty} C_{n k} b_{n} z^{n} \text { and } C_{n k}=\frac{n !}{|n-k| !} .
$$

In this paper, motivated by study in [9], a new class $A_{H}(k, \alpha, \gamma)\left(k \in \mathbb{N}_{0}=\mathbb{N} \cup\{0\}, 0 \leq \gamma \leq 1\right.$, $0 \leq \alpha<1$, ) of harmonic univalent functions in $U=\{z \in \mathbb{C}:|z|<1\}$ is introduced and studied. Furthermore, coefficient conditions, distortion bounds, extreme points, convex combination and radii of convexity for this class are obtained.

\section{Main Results}

\subsection{The Class $A_{H}(k, \alpha, \gamma)$}

Definition 1. Let $f(z)=h(z)+\overline{g(z)}$ be a harmonic function, where $h(z)$ and $g(z)$ are given by Equation (1). Thenf $(z) \in A_{H}(k, \alpha, \gamma)$ it satisfies

$$
\operatorname{Re}\left\{\frac{F^{k+1} f(z)}{(1-\gamma) z+\gamma F^{k} f(z)}\right\}>\alpha,
$$

fork $\in \mathbb{N}_{0}=\mathbb{N} \cup\{0\}, 0 \leq \gamma \leq 1,0 \leq \alpha<1, z \in U$, and $F^{k} f(z)$ defined by Equation (4)

Let $A_{\bar{H}}(k, \alpha, \gamma)$ be the subclass of $A_{H}(k, \alpha, \gamma)$, where $A_{\bar{H}}(k, \alpha, \gamma)=S_{\bar{H}} \cap A_{H}(k, \alpha, \gamma)$.

Remark 1. The class $A_{\bar{H}}(k, \alpha, \gamma)$ reduces to the class $B_{\bar{H}}(k, \alpha)[9]$, when $\gamma=1$.

Here, we give a sufficient condition for a function $f$ to be in the class $A_{H}(k, \alpha, \gamma)$.

Theorem 1. Let $f(z)=h(z)+\overline{g(z)}$ where $h(z)$ and $g(z)$ were given by (1). If

$$
\sum_{n=2}^{\infty} \varnothing(n, k, \alpha, \gamma)\left|a_{n}\right|+\sum_{n=1}^{\infty} \psi(n, k, \alpha, \gamma)\left|b_{n}\right| \leq 1
$$


where

$$
\begin{gathered}
\varnothing(n, k, \alpha, \gamma)=\frac{(|n-k|-\alpha \gamma) C_{n k}}{(1-\alpha)} \\
\psi(n, k, \alpha, \gamma)=\frac{(|n-k|+\alpha \gamma) C_{n k}}{(1-\alpha)} \\
\left(k \in \mathbb{N}_{0}=\mathbb{N} \cup\{0\}, 0 \leq \gamma \leq 1,0 \leq \alpha<1, n \in \mathbb{N}\right),
\end{gathered}
$$

then $f(z)$ is harmonic univalent and sense-preserving in $U$ and $f(z) \in A_{H}(k, \alpha, \gamma)$.

Proof. Firstly, to show that $f(z)$ is harmonic univalent in $U$, suppose that $z_{1}, z_{2} \in U$ for $\left\lfloor z_{1}\right\rfloor \leq\left\lfloor z_{2}\right\rfloor<1$, we have by inequality so that $z_{1} \neq z_{2}$, then

$$
\begin{aligned}
\left|\frac{f\left(z_{1}\right)-f\left(z_{2}\right)}{h\left(z_{1}\right)-h\left(z_{2}\right)}\right| & \\
& \geq 1-\left|\frac{g\left(z_{1}\right)-g\left(z_{2}\right)}{h\left(z_{1}\right)-h\left(z_{2}\right)}\right|=1-\left|\frac{\sum_{n=1}^{\infty} b_{n}\left(z_{1}^{n}-z_{2}^{n}\right)}{\left(z_{1}-z_{2}\right)-\sum_{n=2}^{\infty} a_{n}\left(z_{1}^{n}-z_{2}^{n}\right)}\right| \\
& \geq 1-\frac{\sum_{n=1}^{\infty} n\left|b_{n}\right|}{1-\sum_{n=2}^{\infty}\left|a_{n}\right|} \geq 1-\frac{\sum_{n=1}^{\infty} \frac{(|n-k|+\alpha \gamma) C_{n k}}{(1-\alpha)}\left|b_{n}\right|}{1-\sum_{n=2}^{\infty} \frac{(|n-k|-\alpha \gamma) C_{n k}}{(1-\alpha)}\left|a_{n}\right|} \geq 0 .
\end{aligned}
$$

Thus $f$ is a univalent function in $U$.

Note that $f$ is sense-preserving in $U$. This is because

$$
\begin{aligned}
\left|h^{\prime}(z)\right| \geq & 1-\sum_{n=2}^{\infty} n\left|a_{n}\right||z|^{n-1}>1-\sum_{n=2}^{\infty} n\left|a_{n}\right| \geq 1-\sum_{n=2}^{\infty} \frac{(|n-k|-\alpha \gamma) C_{n k}}{(1-\alpha)}\left|a_{n}\right| \\
& \geq \sum_{n=1}^{\infty} \frac{(|n-k|+\alpha \gamma) C_{n k}}{(1-\alpha)}\left|b_{n}\right| \geq \sum_{n=1}^{\infty} n\left|b_{n}\right| \geq \sum_{n=1}^{\infty} n\left|b_{n}\right||z|^{n-1} \geq\left|g^{\prime}(z)\right| .
\end{aligned}
$$

According to the condition of Equation (5), we only need to show that if Equation (6) holds, then

$$
\operatorname{Re}\left\{\frac{F^{k+1} f(z)}{(1-\gamma) z+\gamma F^{k} f(z)}\right\}=\operatorname{Re}\left(w=\frac{A(z)}{B(z)}\right)>\alpha
$$

where $z=r e^{i \theta}, 0 \leq \theta \leq 2 \pi, 0 \leq r<1$ and $0 \leq \alpha<1$.

Note that $A(z)=F^{k+1} f(z)$ and $B(z)=(1-\gamma) z+\gamma F^{k} f(z)$.

Using the fact that $\operatorname{Re}(w)>\alpha$ if and only if $|w-(1+\alpha)| \leq|w+(1-\alpha)|$, it suffices to show that

$$
|A(z)-(1+\alpha) B(z)|-|A(z)+(1-\alpha) B(z)| \leq 0
$$

Substituting for $A(z)$ and $B(z)$ in $|A(z)-(1+\alpha) B(z)|$, we obtain

$$
\begin{aligned}
|A(z)-(1+\alpha) B(z)|= & \left|F^{k+1} f(z)-(1+\alpha)\left[(1-\gamma) z+\gamma F^{k} f(z)\right]\right| \\
= & \mid\left[z+\sum_{n=2}^{\infty} C_{n(k+1)} a_{n} z^{n}+(-1)^{(k+1)} \sum_{n=1}^{\infty} C_{n(k+1)} \overline{b_{n} z^{n}}\right] \\
& -(1+\alpha)\left[(1-\gamma) z+\gamma z+\gamma \sum_{n=2}^{\infty} C_{n k} a_{n} z^{n}+\gamma(-1)^{k} \sum_{n=1}^{\infty} C_{n k} \overline{b_{n} z^{n}}\right] \mid \\
\leq & \alpha|z|+\sum_{n=2}^{\infty}|(\gamma(1+\alpha))-| n-k|| C_{n k}\left|a_{n}\right||z|^{n} \\
& +\sum_{n=1}^{\infty}|(\gamma(1+\alpha))+| n-k|| C_{n k}\left|a_{n}\right||\bar{z}|^{n} .
\end{aligned}
$$


Now, substituting for $A(z)$ and $B(z)$ in $|A(z)+(1-\alpha) B(z)|$, we obtain

$$
\begin{aligned}
& |A(z)+(1-\alpha) B(z)|=\left|F^{k+1} f(z)+(1-\alpha)\left[(1-\gamma) z+\gamma F^{k} f(z)\right]\right| \\
& =\left\lfloor\left[z+\sum_{n=2}^{\infty} C_{n(k+1)} a_{n} z^{n}+(-1)^{(k+1)} \sum_{n=1}^{\infty} C_{n(k+1)} \overline{b_{n} z^{n}}\right\rfloor\right. \\
& +(1-\alpha)\left[(1-\gamma) z+\gamma z+\gamma \sum_{n=2}^{\infty} C_{n k} a_{n} z^{n}+\gamma(-1)^{k} \sum_{n=1}^{\infty} C_{n k} \overline{b_{n} z^{n}}\right] \mid \\
& \geq(2-\alpha)|z|-\sum_{n=2}^{\infty}|(\gamma(\alpha-1))-| n-k|| C_{n k}\left|a_{n}\right||z|^{n} \\
& -\sum_{n=1}^{\infty}|| n-k|-(\gamma(1-\alpha))| C_{n k}\left|a_{n}\right||\bar{z}|^{n} \text {. }
\end{aligned}
$$

Substituting for Equations (8) and (9) in the inequality we obtain

$$
\begin{aligned}
& |A(z)-(1+\alpha) B(z)|-|A(z)+(1-\alpha) B(z)| \\
& \leq \alpha|z|+\sum_{n=2}^{\infty}|(\gamma(1+\alpha))-| n-k|| C_{n k}\left|a_{n}\right||z|^{n} \\
& \quad+\sum_{n=1}^{\infty}|(\gamma(1+\alpha))+| n-k|| C_{n k}\left|b_{n}\right||\bar{z}|^{n} \\
& \quad+(\alpha-2)|Z|+\sum_{n=2}^{\infty}|(\gamma(\alpha-1))-| n-k|| C_{n k}\left|a_{n}\right||z|^{n} \\
& \quad+\sum_{n=1}^{\infty}|| n-k|-(\gamma(1-\alpha))| C_{n k}\left|b_{n}\right||\bar{z}|^{n} . \\
& =2 \sum_{n=2}^{\infty}(|n-k|-\alpha \gamma) C_{n k}\left|a_{n}\right|+2 \sum_{n=1}^{\infty}(|n-k|+\alpha \gamma) C_{n k}\left|b_{n}\right|-2(1-\alpha) \\
& \quad \leq 0 . \text { (by hypothesis). }
\end{aligned}
$$

Therefore, we have

$$
\sum_{n=2}^{\infty}(|n-k|-\alpha \gamma) C_{n k}\left|a_{n}\right|+\sum_{n=1}^{\infty}(|n-k|+\alpha \gamma) C_{n k}\left|b_{n}\right| \leq(1-\alpha)
$$

The harmonic univalent function

$$
f(z)=z+\sum_{n=2}^{\infty} \frac{1}{\varnothing(n, k, \alpha, \gamma)} \mathcal{X}_{n} z^{n}+\sum_{n=1}^{\infty} \frac{1}{\psi(n, k, \alpha, \gamma)} \overline{\mathcal{Y}_{n} z^{n}},
$$

where $k \in \mathbb{N}_{0}$ and $\sum_{k=2}^{\infty}\left|\mathcal{X}_{n}\right|+\sum_{k=1}^{\infty}\left|\mathcal{Y}_{n}\right|=1$, shows that the coefficient bound given by Equation (6) is sharp. Since

$$
\begin{aligned}
& \sum_{n=2}^{\infty} \varnothing(n, k, \alpha, \gamma)\left|a_{n}\right|+\sum_{n=1}^{\infty} \psi(n, k, \alpha, \gamma)\left|b_{n}\right| \\
& =\sum_{n=2}^{\infty} \varnothing(n, k, \alpha, \gamma) \frac{1}{\varnothing(n, k, \alpha, \gamma)}\left|\mathcal{X}_{n}\right|+\sum_{n=1}^{\infty} \psi(n, k, \alpha, \gamma) \frac{1}{\psi(n, k, \alpha, \gamma)}\left|\mathcal{Y}_{n}\right| \\
& =\sum_{n=2}^{\infty}\left|\mathcal{X}_{n}\right|+\sum_{n=1}^{\infty}\left|\mathcal{Y}_{n}\right|=1 .
\end{aligned}
$$

Now, we show that the condition of Equation (6) is also necessary for functions $f_{k}=h+\overline{g_{k}}$, where $h$ and $g_{n}$ are given by Equation (6).

Theorem 2. Let $f_{k}=h+\overline{g_{k}}$ be given by Equation (6). Then $f_{k}(z) \in A_{\bar{H}}(k, \alpha, \gamma)$ if and only if the coefficient in condition of Equation (6) holds. 
Proof. We only need to prove the "only if" part of the theorem because of $A_{\bar{H}}(k, \alpha, \gamma) \subset A_{H}(k, \alpha, \gamma)$. Then by Equation (5), we have

$$
\operatorname{Re}\left\{\frac{F^{k+1} f(z)}{(1-\gamma) z+\gamma F^{k} f(z)}\right\}>\alpha
$$

or, equivalently

$$
\operatorname{Re}\left[\frac{\begin{array}{c}
z-\sum_{n=2}^{\infty} C_{n(k+1)}\left|a_{n}\right| z^{n}+(-1)^{2 k+1} \sum_{n=1}^{\infty} C_{n(k+1)}\left|b_{n}\right| \bar{z}^{n} \\
-\alpha\left\{(1-\gamma) z+\gamma z+\gamma \sum_{n=2}^{\infty} C_{n k}\left|a_{n}\right| z^{n}+\gamma(-1)^{2 k} \sum_{n=1}^{\infty} C_{n k}\left|b_{n}\right| \bar{z}^{n}\right\}
\end{array}}{(1-\gamma) z+\gamma z-\gamma \sum_{n=2}^{\infty} C_{n k}\left|a_{n}\right| z^{n}+\gamma(-1)^{2 k} \sum_{n=1}^{\infty} C_{n k}\left|b_{n}\right| \bar{z}^{n}}\right] \geq 0
$$

We observe that the above-required condition of Equation (11) must behold for all values of $z$ in $U$. If we choose $z$ to be real and $z \rightarrow 1^{-}$, we get

$$
\begin{gathered}
(1-\alpha)-\sum_{n=2}^{\infty}(|n-k|-\alpha \gamma) C_{n k}\left|a_{n}\right| \\
\frac{+\sum_{n=1}^{\infty}(|n-k|+\alpha \gamma) C_{n k}\left|b_{n}\right|}{1-\gamma \sum_{n=2}^{\infty} C_{n k}\left|a_{n}\right| z^{n-1}+\gamma \sum_{n=1}^{\infty} C_{n k}\left|b_{n}\right| \bar{z}^{n-1}} \geq 0
\end{gathered}
$$

If the condition (6) does not hold, then the numerator in Equation (12) is negative for $r$ sufficiently closed to 1 . Hence there exist $z_{0}=r_{0}$ in $(0,1)$ for which the quotient in Equation (12) is negative, therefore there is a contradicts the required condition for $f_{k} \in A_{\bar{H}}(k, \alpha, \gamma)$.

\subsection{Extreme Points}

Here, we determine the extreme points of the closed convex hull of $A_{\bar{H}}(k, \alpha, \gamma)$, denoted by $\operatorname{clco} A_{\bar{H}}(k, \alpha, \gamma)$.

Theorem 3. Let $f_{k}$ given by (1.2). Then $f_{k} \in A_{\bar{H}}(k, \alpha, \gamma)$ if and only if

$$
f_{k}(z)=\sum_{n=1}^{\infty}\left(\mathcal{X}_{n} h_{n}+\mathcal{Y}_{n} g_{k n}\right)
$$

where

$$
\begin{gathered}
h_{1}(z)=z, h_{n}(z)=z-\frac{1}{\varnothing(n, k, \alpha, \gamma)} z^{n}, n=2,3, \ldots, \\
g_{k n}(z)=z+(-1)^{k} \frac{1}{\psi(n, k, \alpha, \gamma)} \bar{z}^{n}, n=1,2, \ldots,
\end{gathered}
$$

and

$$
\mathcal{X}_{n} \geq 0, \mathcal{Y}_{n} \geq 0, \mathcal{X}_{1}=1-\sum_{n=2}^{\infty}\left(\mathcal{X}_{n}+\mathcal{Y}_{n}\right) \geq 0
$$

In particular the extreme points of $A_{\bar{H}}(k, \alpha, \gamma)$ are $\left\{h_{n}\right\}$ and $\left\{g_{k n}\right\}$.

Proof. Suppose

$$
\begin{aligned}
f_{k}(z) & =\sum_{n=1}^{\infty}\left(\mathcal{X}_{n} h_{n}+\mathcal{Y}_{n} g_{k n}\right) \\
& =\sum_{n=1}^{\infty}\left(\mathcal{X}_{n} h_{n}+\mathcal{Y}_{n} g_{k n}\right) z-\sum_{n=2}^{\infty} \frac{1}{\varnothing((n, k, \alpha, \gamma)} \mathcal{X}_{n} z^{n}+(-1)^{k} \sum_{n=1}^{\infty} \frac{1}{\psi(n, k, \alpha, \gamma)} \mathcal{Y}_{n} \bar{z}^{n} \\
& =z-\sum_{n=2}^{\infty} \frac{1}{\varnothing(n, k, \alpha, \gamma)} \mathcal{X}_{n} z^{n}+(-1)^{k-1} \sum_{n=1}^{\infty} \frac{1}{\psi(n, k, \alpha, \gamma)} \mathcal{Y}_{n} \bar{z}^{n}
\end{aligned}
$$


Then

$$
\begin{aligned}
& \sum_{n=2}^{\infty} \varnothing(n, k, \alpha, \gamma)\left|a_{n}\right|+\sum_{n=1}^{\infty} \psi(n, k, \alpha, \gamma)\left|b_{n}\right| \\
& =\sum_{k=2}^{\infty} \varnothing(n, k, \alpha, \gamma)\left(\frac{1}{\varnothing(n, k, \alpha, \gamma)} \mathcal{X}_{n}\right)+\sum_{k=1}^{\infty} \psi(n, k, \alpha, \gamma)\left(\frac{1}{\psi(n, k, \alpha, \gamma)} \mathcal{Y}_{n}\right) \\
& =\sum_{n=2}^{\infty} \mathcal{X}_{n}+\sum_{n=1}^{\infty} \mathcal{Y}_{n}=1-\mathcal{X}_{1} \leq 1 .
\end{aligned}
$$

Therefore $f_{k}(z) \in \operatorname{clco} A_{\bar{H}}(k, \alpha, \gamma)$.

Conversely, if $f_{k}(z) \in \operatorname{clco} A_{\bar{H}}(k, \alpha, \gamma)$. Then

$$
\begin{gathered}
\text { Set } \mathcal{X}_{n}=\varnothing(n, k, \alpha, \gamma)\left|a_{n}\right|,(n=2,3, \ldots) \text { and } \mathcal{Y}_{n}=\Psi(n, k, \alpha, \gamma)\left|b_{n}\right|, \\
(n=1,2, \ldots) \text { and } \mathcal{X}_{1}=1-\sum_{n=2}^{\infty} \mathcal{X}_{n}+\sum_{n=1}^{\infty} \mathcal{Y}_{n}
\end{gathered}
$$

The required representation is obtained as

$$
\begin{aligned}
f_{k}(z) & =z-\sum_{n=2}^{\infty}\left|a_{n}\right| z^{n}+(-1)^{k} \sum_{n=1}^{\infty}\left|b_{n}\right| \bar{z}^{n} \\
& =z-\sum_{n=2}^{\infty} \frac{1}{\varnothing(n, k, \alpha, \gamma)} \mathcal{X}_{n} z^{n}+(-1)^{k} \sum_{n=1}^{\infty} \frac{1}{\psi(n, k, \alpha, \gamma)} \mathcal{Y}_{n} \bar{z}^{n} \\
& =z-\sum_{n=2}^{\infty}\left[z-h_{n}(z)\right] \mathcal{X}_{n}+\sum_{n=1}^{\infty}\left[z-g_{k n}(z)\right] \mathcal{Y}_{n} \\
& =\left[1-\sum_{n=2}^{\infty} \mathcal{X}_{n}-\sum_{n=1}^{\infty} \mathcal{Y}_{n}\right] z+\sum_{n=2}^{\infty} h_{n}(z) \mathcal{X}_{n}+\sum_{n=1}^{\infty} g_{k n}(z) \mathcal{Y}_{n}=\sum_{n=1}^{\infty}\left(\mathcal{X}_{n} h_{n}+\mathcal{Y}_{n} g_{k n}\right)
\end{aligned}
$$

\subsection{Convex Combination}

Here, we show that the class $A_{\bar{H}}(k, \alpha, \gamma)$ is closed under convex combination of its members.

Let the function $f_{k, i}(z)$ be defined, for $i=1,2, \ldots, m$ by

$$
f_{k, i}(z)=z-\sum_{n=2}^{\infty}\left|a_{n, i}\right| z^{n}+(-1)^{k} \sum_{n=1}^{\infty}\left|b_{n, i}\right| \bar{z}^{n}
$$

Theorem 4. Let the functions $f_{k, i}(z)$, defined by Equation (13) be in the class $A_{\bar{H}}(k, \alpha, \gamma)$, for every $i=1,2, \ldots, m$. Then the functions $c_{i}(z)$ defined by

$$
c_{i}(z)=\sum_{i=1}^{\infty} t_{i} f_{k, i}(z), 0 \leq t_{i} \leq 1
$$

are also in the class $A_{\bar{H}}(k, \alpha, \gamma)$, where $\sum_{i=1}^{\infty} t_{i}=1$.

Proof. According to the definition of $c_{i}(z)$, we can write

$$
c_{i}(z)=z-\sum_{n=2}^{\infty}\left(\sum_{i=1}^{\infty} t_{i}\left|a_{n, i}\right|\right) z^{n}+(-1)^{k} \sum_{n=1}^{\infty}\left(\sum_{i=1}^{\infty} t_{i}\left|b_{n, i}\right|\right) \bar{z}^{n}
$$


Further, since $f_{k, i}(z)$ are in $A_{\bar{H}}(k, \alpha, \gamma)$ for every $i=1,2, \ldots, m$, then by Theorem 2 , we obtain

$$
\begin{aligned}
& \sum_{k=2}^{\infty} \varnothing(n, k, \alpha, \gamma)\left(\sum_{i=1}^{\infty} t_{i}\left|a_{n, i}\right|\right)+\sum_{k=1}^{\infty} \psi(n, k, \alpha, \gamma)\left(\sum_{i=1}^{\infty} t_{i}\left|b_{n, i}\right|\right) \\
& =\sum_{i=1}^{\infty} t_{i}\left(\sum_{k=2}^{\infty} \varnothing(n, k, \alpha, \gamma)\left|a_{n, i}\right|+\sum_{k=1}^{\infty} \psi(n, k, \alpha, \gamma)\left|b_{n, i}\right|\right) \leq \sum_{i=1}^{\infty} t_{i}=1,
\end{aligned}
$$

which is required coefficient condition.

\subsection{Convolution (Hadamard Product) Property}

Here, we show that the class $A_{\bar{H}}(k, \alpha, \gamma)$ is closed under convolution.

The convolution of two harmonic functions

$$
f_{k}(z)=z-\sum_{n=2}^{\infty}\left|a_{n}\right| z^{n}+(-1)^{k} \sum_{n=1}^{\infty}\left|b_{n}\right| \bar{z}^{n}
$$

and

$$
Q_{n}(z)=z-\sum_{n=2}^{\infty}\left|L_{n}\right| z^{n}+(-1)^{k} \sum_{n=1}^{\infty}\left|M_{n}\right| \bar{z}^{n}
$$

is defined as

$$
\begin{gathered}
\left(f_{n} * Q_{n}\right)(z)=f_{n}(z) * Q_{n}(z) \\
=z-\sum_{n=2}^{\infty}\left|a_{n} L_{n}\right| z^{n}+(-1)^{k} \sum_{n=1}^{\infty}\left|b_{n} M_{n}\right| \bar{z}^{n}
\end{gathered}
$$

Using Equations (12)-(14), we prove the following theorem.

Theorem 5. For $0 \leq \mu \leq \alpha<1, k \in \mathbb{N}_{0}$, let $f_{n} \in A_{\bar{H}}(k, \alpha, \gamma)$ and $Q_{n} \in A_{\bar{H}}(k, \mu, \gamma)$. Then

$$
f_{n} * Q_{n} \in A_{\bar{H}}(k, \alpha, \gamma) \subset A_{\bar{H}}(k, \mu, \gamma)
$$

Proof. Let

$$
f_{k}(z)=z-\sum_{n=2}^{\infty}\left|a_{n}\right| z^{n}+(-1)^{k} \sum_{n=1}^{\infty}\left|b_{n}\right| \bar{z}^{n}
$$

be in the class $A_{\bar{H}}(k, \alpha, \gamma)$ and

$$
Q_{n}(z)=z-\sum_{n=2}^{\infty}\left|L_{n}\right| z^{n}+(-1)^{k} \sum_{n=1}^{\infty}\left|M_{n}\right| \bar{z}^{n}
$$

be in $A_{\bar{H}}(k, \mu, \gamma)$.

Then the convolution $f_{n} * Q_{n}$ is given by Equation (16), we want to show that the coefficients of $f_{n} * Q_{n}$ satisfy the required condition given in Theorem 1 .

For $Q_{n} \in A_{\bar{H}}(k, \mu, \gamma)$, we note that $\left|L_{n}\right|<1$ and $\left|M_{n}\right|<1$. Now consider convolution functions $f_{n} * Q_{n}$ as follows:

$$
\begin{aligned}
& \sum_{k=2}^{\infty} \varnothing(n, k, \mu, \gamma)\left|a_{n}\right|\left|L_{n}\right|+\sum_{k=1}^{\infty} \psi(n, k, \mu, \gamma)\left|b_{n}\right|\left|M_{n}\right| \\
& \leq \sum_{k=2}^{\infty} \varnothing(n, k, \mu, \gamma)\left|a_{n}\right|+\sum_{k=1}^{\infty} \psi(n, k, \mu, \gamma)\left|b_{n}\right| \leq 1 .
\end{aligned}
$$

Since $0 \leq \mu \leq \alpha<1$ and $f_{n} \in A_{\bar{H}}(k, \alpha, \gamma)$. Therefore $f_{n} * Q_{n} \in A_{\bar{H}}(k, \alpha, \gamma) \subset A_{\bar{H}}(k, \mu, \gamma)$. 


\subsection{Integral Operator}

Here, we examine the closure property of the class $A_{\bar{H}}(k, \alpha, \gamma)$ under the generalized Bernardi-Libera-Livingston integral operator (see References $[10,11]) \mathcal{L}_{u}(f)$ which is defined by,

$$
\mathcal{L}_{u}(f)=\frac{u+1}{z^{u}} \int_{0}^{z} t^{u-1} f(t) d t, u>-1 .
$$

Theorem 6. Let $f_{k}(z) \in A_{\bar{H}}(k, \alpha, \gamma)$. Then

$$
\mathcal{L}_{u}\left(f_{k}(z)\right) \in A_{\bar{H}}(k, \alpha, \gamma)
$$

Proof. From definition of $\mathcal{L}_{u}\left(f_{k}(z)\right)$ given by Equation (17), it follows that

$$
\begin{aligned}
\mathcal{L}_{u}\left(f_{k}(z)\right) & =\frac{u+1}{z^{u}} \int_{0}^{z} t^{u-1}\left(t-\sum_{n=2}^{\infty}\left|a_{n}\right| t^{n}+(-1)^{k} \sum_{n=1}^{\infty}\left|b_{n}\right| t^{n}\right) d t \\
& =z-\sum_{n=2}^{\infty} \frac{u+1}{u+n}\left|a_{n}\right| z^{n}+(-1)^{k} \sum_{n=1}^{\infty} \frac{u+1}{u+n}\left|b_{n}\right| z^{n} \\
& =z-\sum_{n=2}^{\infty} G_{n} z^{n}+(-1)^{n-1} \sum_{n=1}^{\infty} L_{n} z^{n}
\end{aligned}
$$

where

$$
\begin{gathered}
G_{n}=\frac{u+1}{u+n}\left|a_{n}\right|, \text { and } \\
L_{n}=\frac{u+1}{u+n}\left|b_{n}\right|
\end{gathered}
$$

Hence

$$
\begin{aligned}
& \sum_{k=2}^{\infty} \varnothing(n, k, \alpha, \gamma) \frac{u+1}{u+n}\left|a_{n}\right|+\sum_{k=1}^{\infty} \psi(n, k, \alpha, \gamma) \frac{u+1}{u+n}\left|b_{n}\right| \\
& \leq \sum_{n=2}^{\infty} \varnothing(n, k, \alpha, \gamma)\left|a_{n}\right|+\sum_{n=1}^{\infty} \psi(n, k, \alpha, \gamma)\left|b_{n}\right| \leq 1 .
\end{aligned}
$$

by Theorem 2 .

Therefore, we have $\mathcal{L}_{u}\left(f_{k}(z)\right) \in A_{\bar{H}}(k, \alpha, \gamma)$.

Author Contributions: Conceptualization, methodology, validation, formal analysis, investigation, writing —original draft preparation, writing-review and editing, A.K.A.-k.; visualization and supervision, W.G.A.; project administration and supervision, S.S.A.

Funding: This research received no external funding

Conflicts of Interest: The authors declare no conflict of interest.

\section{References}

1. Clunie, J.; Sheil, S.T. Harmonic Univalent functions. Ann. Acad. Sci. Fenn. Ser. A I. Math. 1984, 9, 3-25. [CrossRef]

2. Bernardi, S.D. Convex and Starlike Univalent Function. Trans. Am. Math. Soc. 1969, 135, 429-446. [CrossRef]

3. Dixit, K.K.; Pathak, A.L.; Porwal, S.; Agarwal, R. On a Subclass of Harmonic Univalent functions defined by Convolution and Integral Convolution. Int. J. Pure Appl. Math. 2011, 63, 255-264.

4. Bhaya, E.S.; Kareem, M.A. Whitney multi approximation. J. Univ. Babylon Pure Appl. Sci. 2016, 7, 2395-2399.

5. Bhaya, E.S.; Almurieb, H.A. Neural network trigonometric approximation. J. Univ. Babylon Pure Appl. Sci. 2018, 7, 385-403. [CrossRef]

6. Makinde, D.O.; Afolabi, A.O. On a Subclass of Harmonic Univalent Functions. Trans. J. Sci. Technol. 2012, 2,1-11.

7. Porwal, S.; Shivam, K. A New subclass of Harmonic Univalent functions defined by derivative operator. Electron. J. Math. Anal. Appl. 2017, 5, 122-134. 
8. Makinde, D.O. On a new Differential Operator. Theor. Math. Appl. 2016, 6, 71-74.

9. Sharma, R.B.; Ravindar, B. On a subclass of harmonic univalent functions. J. Phys. Conf. Ser. 2018, 1000, 012115. [CrossRef]

10. Bharavi, S.R.; Haripriya, M. On a class of $\alpha$-convex functions subordinate to a shell-shaped region. J. Anal. 2017, 25, 99-105.

11. Libera, R.J. Some Classes of Regular Univalent Functions. Proc. Am. Math. Soc. 1965, 16, 755-758. [CrossRef] 